\title{
A Study of Emotion Recognition for Constructive Learning using Robots
}

\author{
Veena Vijayan V. \\ Dept of Computer Science, \\ Mar Baselios College of \\ Engineering and Technology \\ Trivandrum, India
}

\begin{abstract}
The robot starts to play a prominent role in the modern world. They can be used in different areas like house hold activities, markets, military purposes, medical fields and schools. The main aim of this paper is to focus the use of robot in the educational field. Better feedback will be obtained through efficient human robot interactions. Human robot interaction defines the ability of the robot to interact efficiently with humans. The robot should monitor the student and analyze the emotions dynamically. The main aim is to maintain a healthy learning rate after recognizing the positive emotions. Real time face tracking and feature extraction is applied to recognize the emotional state of the student.
\end{abstract}

\section{General Terms}

Constructive Learning, Face and Feature extraction, 3-D wire frame model, Tree Augmented Naïve Bayes classifier.

\section{Keywords}

Face tracking, Action units, Bezier volume, Real time recognition.

\section{INTRODUCTION}

Human face can show a wide variety of emotions. The emotions can be negative or positive. The positive emotions include happiness, surprise, pleasant and negative emotions include anger, unpleasant, sad, fear. Skilled humans can capture these emotions with varying degrees of accuracy. The recognition of these emotions helps the teacher to take necessary corrective measures to increase the effectiveness of the class. Many years ago researchers were trying to develop computers or some other computer controlled devices that are capable of the similar abilities like recognizing expressions. Although computers perform as good as or better than people in different domains, they do not yet rise to human levels of monitoring [5].

\section{RELATED WORK}

\subsection{Educational robots for Children}

The e-Learning is expected to bring a revolution to the education system by providing different opportunities to share abundant information and knowledge [2]. IROBI was the human friendly Intelligent home Robot developed under the eLearning technology that provides home monitoring, entertainment, messaging, home tutoring and security. They provide facial expressions like happiness, calm and sadness. In comparison with other Medias, these robots make differences in the effect of learning [2]. Most of the children reacted to the motions and facial expression of the IROBI. Figure 1 [18] shows the interaction between the IROBI and the child.

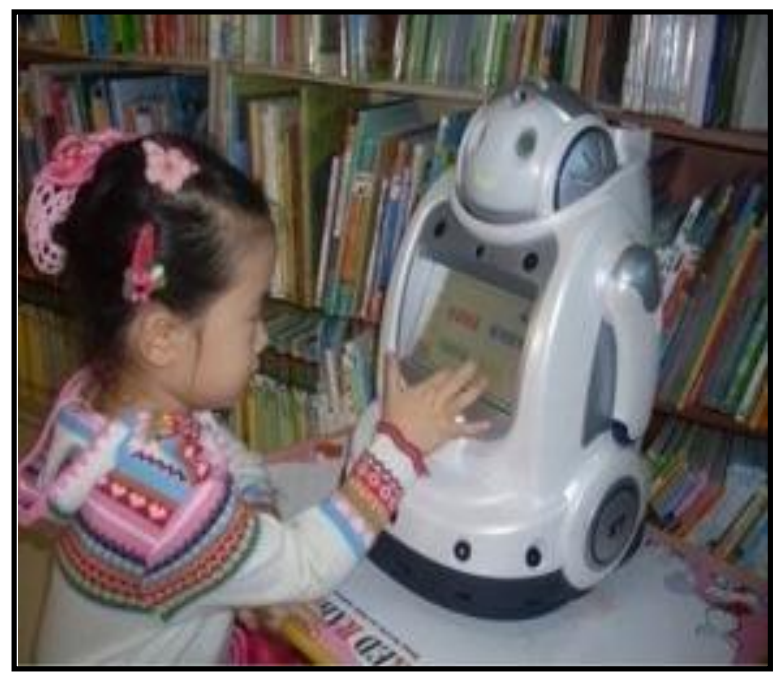

Figure 1. Child interacting with the IROBI [18]

It also features auto travelling to caller, emotional expressions, automatic charging, online update of contents and voice recognition. IROBI has the following specifications [2].

- Ram:256 Mbytes

- Hard disk drive :30 Gbytes

- Power consumption :75 w

- Charging time :3 hours

- Running time :3 hours(off)/10 hours(on)

- Speed: maximum $25 \mathrm{~cm} / \mathrm{sec}$

- $\quad$ Size:650(H)*430(W)*480(D) in mm

- Lan :wireless Lan 10 mbps

- Cam:33k pixel CMOS cam

- Battery :9000mAh

- Sensors: human sensing, floor detection, ultrasonic

Results suggested that robots were more effective to children's learning interest, concentration and academic achievement. These implications introduce robot in the field of constructive learning.

\section{TOWARDS CONSTRUCTIVE LEARNING}

Constructive learning is a learning process in which learning rate of student is enhanced through critical thinking and motivation. This type of learning improves the interactive 
communication and social skills among the students. Traditional class rooms systems enhance the learning activities through repetition, strict adherence and guidance from the textbook which contradicts the ideas of constructive learning. Emotional states of the humans can be classified into positive and negative. Positive emotions provide a novel perspective to the healthy learning. Negative emotions will reduce the learning rate and may sometimes lead to the restarting of the whole learning process. The relationship between learning and emotions is graphically represented in figure 2 . The area above the horizontal axis shows the constructive learning and below shows the un-learning. The emotional state is observed to be negative if there is a shift from I to II or III quadrant. So the main aim of the instructor is to analyze the emotional state of the students carefully and should try to move his/her emotional state back to I quadrant [1].

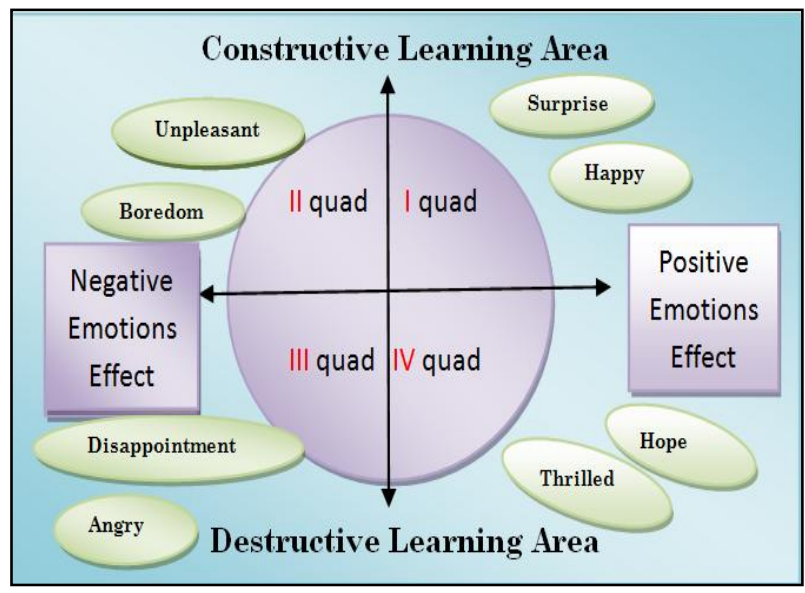

Figure 2. Relationship between emotions and learning

Once the classification is over next step is to recognize these emotions from the human's face using face and feature tracking methods. The main face and feature tracking methods discussed in this paper are Real Time Recognition using Layered Neural Network and 3-D Wire Frame Model using Bezier volume.

\subsection{Constructive learning using robots}

The field of robotics should be exploited to a larger extent for the betterment of educational field. Robotic activities are considered as valuable tools to enhance the problem solving skill, team work skills, communication skills, social skills, creativity and imagination. Home robot embodying e-Learning technologies provides voice, gestures and multimedia contents. It was investigated that significance and effectiveness of the robot as a learning tool provide more effectiveness compared to other web based interaction or audio tapes. This leads to the idea of constructive learning using robots. The robots should monitor the students and observes the emotional transitions of the students from positive state (constructive learning) to the negative state (un- learning).

\subsection{Steps in Constructive learning}

Step1: The robot mimics the actions of the tutor inside the class.

Step2: After the initial corrective action is taken by tutor, emotions of students are recognized by robot using face and feature tracking algorithm.

Step3: The emotions are successfully transferred to the robotic face using actuators.
Step4: Movement of the robot, by analyzing the facial expression of the students.

Step 5: Secondary corrective actions will be taken by the tutor after evaluating the movement of the robot.

\section{FACE AND FEATURE TRACKING}

A real time face tracking and feature extraction is applied to recognize the emotional state of the student. This section deals with the two different technologies for recognizing real time emotions. The emotions under consideration are happiness, fear, anger, disgust, surprise and sadness.

\subsection{Real time recognition using layered neural network}

Face image data of full human face is obtained by using charge coupled camera which has $18 \mathrm{~mm}$ focus distance and resolution of $256 \times 240$ pixels. Real time face recognition using neural network have the following steps [3].

Step1: Brightness distribution of pixels along a vertical line segment crossing the eye and eyebrow region is considered for determining the position of irises.

Step 2: By using center position of irises the position of eyebrow, eyes and mouth is examined. Figure 3 [20] shows the position of eyebrows, eye with respect to the position of irises.

Step 3: Select 13 vertical segments crossing eye, eye-brow, lower and upper lips.

Step 4: Obtain the change in the brightness distribution of these vertical lines and is normalized.

Step 5: This is given as the input to the trained neural network and the recognition of various expressions is the result.

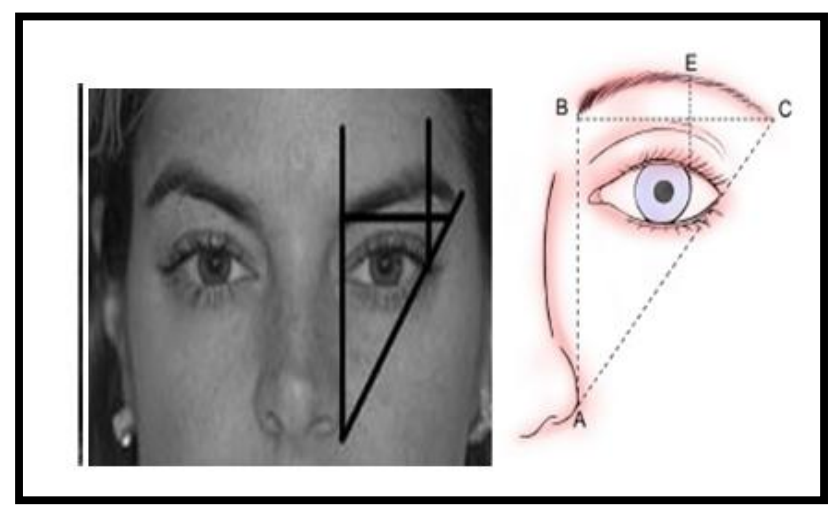

Figure 3. The position of eyebrow, eyes and mouth with respect to the position of irises [20]

For training, different combinations of facial expressions are combined to form action units.

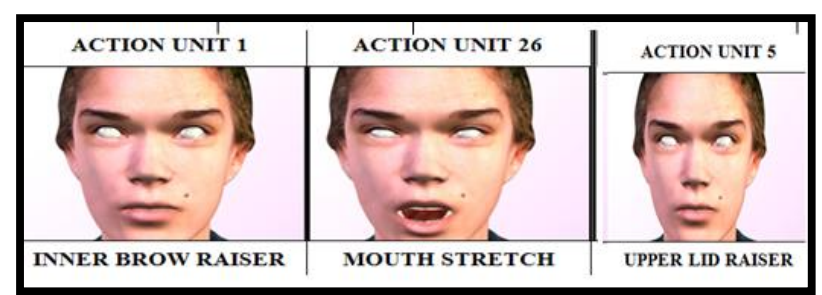


Figure 4. Surprise $=A U$ 1+AU 26+ AU 5

[19]

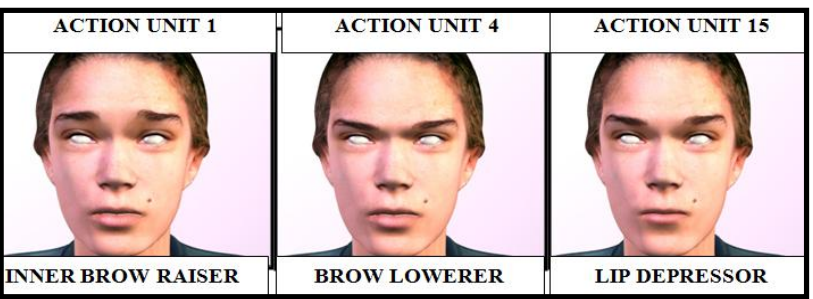

Figure 5. Sad=AU 1+AU 4+AU 15

[19]

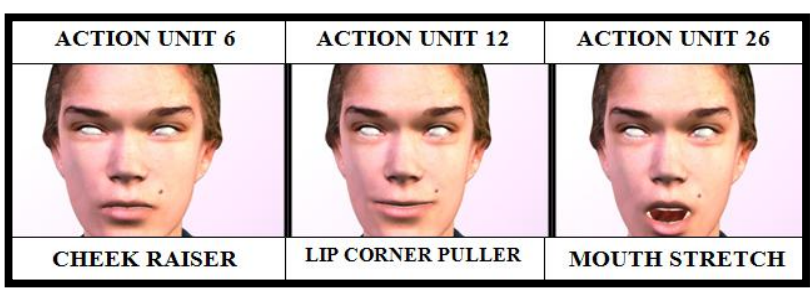

Figure 6. Happy=AU 6+AU 12+AU 26

[19]
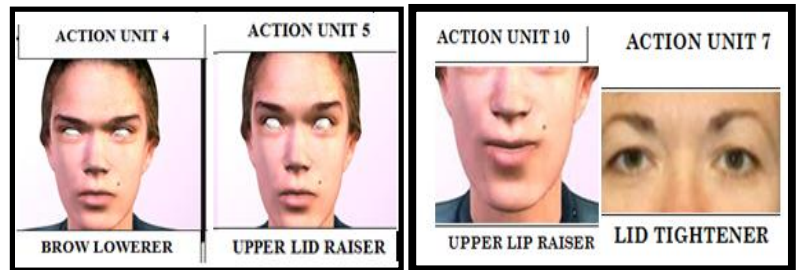

Figure 7. Anger=AU 4+AU $5+$ AU 10+AU 7

[19]

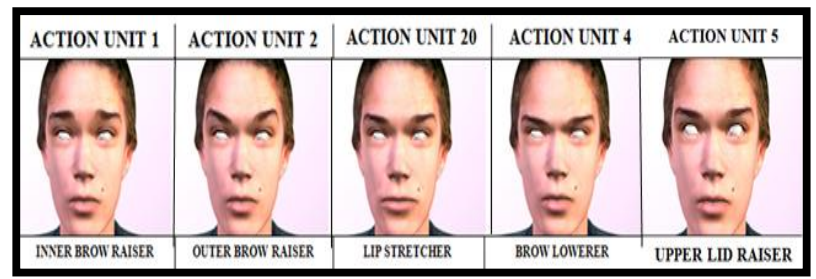

Figure 8. Fear=AU 1+AU 2+AU 20+AU 4+AU 5

[19]

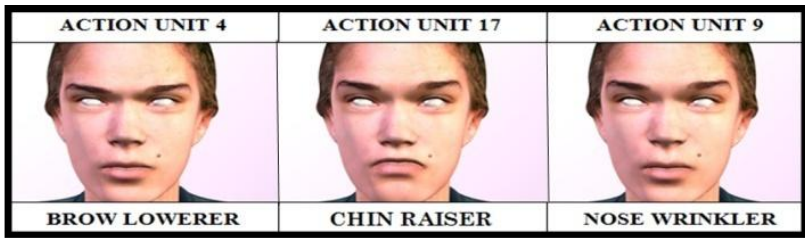

Figure 9. Disgust $=\mathrm{AU}$ 4+AU 17+AU 9

A particular facial expression is designed by the combination of action points. Figures 4 to 9 show the different combination of action units for six basic facial expressions. The displacement magnitude for these control points is determined and micro actuator that has high precision is employed to pull these control points. Finally the emotions are displayed on the face of the robot.

\subsection{Face tracking and feature extraction using 3D wireframe model}

Here a 3-D wire frame model of a face is constructed using the Bezier volume. 3 D Bezier volume model is capable for animating geometric facial models of different shapes and structures. 3-D Bezier volume is defined using the following formula

$$
\mathbf{f}(\mathbf{x}, \mathrm{y}, \mathrm{z})=\sum_{i=0}^{n} \sum_{j=0}^{m} \sum_{k=0}^{l} b_{\mathrm{ijk}} \mathbf{B}_{\mathrm{i}^{\mathrm{n}}}(\mathbf{x}) * \mathbf{B}_{\mathrm{i}^{\mathrm{m}}}(\mathbf{y}) * \mathbf{B}_{\mathrm{i}}{ }^{1}(\mathrm{z})
$$

Face tracking and feature extraction using $3 \mathrm{D}$ wire frame model have the following steps [3].

Step 1: Consider a point inside the volume having the value $\mathrm{f}(\mathrm{x}, \mathrm{y}, \mathrm{z})$ in which $\mathrm{x}, \mathrm{y}, \mathrm{z}$ are parameters in the range 0 to 1 .

Step 2: While considering $B_{i}{ }^{n}(x), B_{i}{ }^{m}(y), B_{i}{ }^{l}(z)$ as the polynomials, control points ' $b_{i j k}$ ' for Bezier surface is selected

Step 3: Multiple Bezier volumes are generated.

Step 4: The movement of facial points such as eye corners and mouth corners is observed by recording the change in control point's $b_{\mathrm{ijk}}$.

Step 5: Then change in each control point $b_{\mathrm{ijk}}$ is calculated as displacement $\mathrm{d}_{\mathrm{ijk}}$, which is important to compute the movement of facial points that determines the emotional state [1]. It can be written as follows:-

$$
\mathrm{S}(\mathrm{x}, \mathrm{y}, \mathrm{z})=\sum_{i=0}^{n} \sum_{j=0}^{m} \sum_{k=0}^{l} d_{\mathrm{ijk}} \mathbf{B}_{\mathrm{i}^{\mathrm{n}}}(\mathbf{x}) * \mathbf{B}_{\mathrm{i}^{\mathrm{m}}}(\mathbf{y}) * \mathrm{~B}_{\mathrm{i}} \mathbf{l}(\mathrm{z})
$$

Step 6: Displacements of the facial points is then programmed as a product of $S$ and $D$ where $S$ is the matrix containing the Bernstein polynomials and $\mathrm{D}$ is the matrix containing the displacement vectors of control point $b_{i j k}$ in MATLAB [1]. A Tree Augmented Naive Bayes classifier is used to recognize the emotional states. The algorithm has following steps:-

Step1: Compute class conditional pair wise mutual information for each pair of features say $\left(\mathrm{Y}_{\mathrm{i}}, \mathrm{Y}_{\mathrm{j}}\right)$.

Step 2: Consider each vertex as a variable.

Step 3: Construct a complete undirected graph.

Step 4: Assign the mutual information computed in step 1 as the weight of each edge.

Step 5: Using Kruskal's algorithm, construct an undirected maximum weighted spanning tee

Step6: Convert the output of step 5 to a directed graph by choosing a root node.

Step 7: Point all the arrows of all edges away from the root.

Step 8: Assign the class node as parent of all feature nodes in step 6 and step 7

\section{CORRECTIVE MEASURES USING ROBOTIC ACTIONS}

Consider a situation where a human instructor is lecturing the students. Once the robot is trained for face tracking and feature extraction, it is placed inside the classroom for real time monitoring. Robot will mimic the actions performed by the instructor with the help of wearable sensors. During the initial stage the emotional state of the student will be positive and hence lies on the first quadrant. After some period the instructor observes a shift in the emotional state of the students 
from the motivated state to confused state. The following steps should be carried out to maintain a healthy learning rate [1].

Step 1: Instructor observes the emotional transition from positive to negative.

Step 2: Initial corrective action is taken by the instructor by repeating the topic.

Step 3: This time the robot stops mimicking and monitors the student.

Step 4: If the initial corrective actions were successful then performs step 5 else step 7 .

Step 5: Robot turns left.

Step 6: Student emotional state shift to the first (motivated) quadrant.

Step 7: Student emotional state shift to III quadrant.

Step 8: Intensified corrective actions by the tutor to make the transition of emotional state to the I quadrant.

Step 9: If the Robot turns left twice, then perform step 6 else step 10

Step 10: Restart the whole learning process.

The Figure 10 shows the overall schematic of the corrective actions taken by the instructor after analyzing the robotic movements.

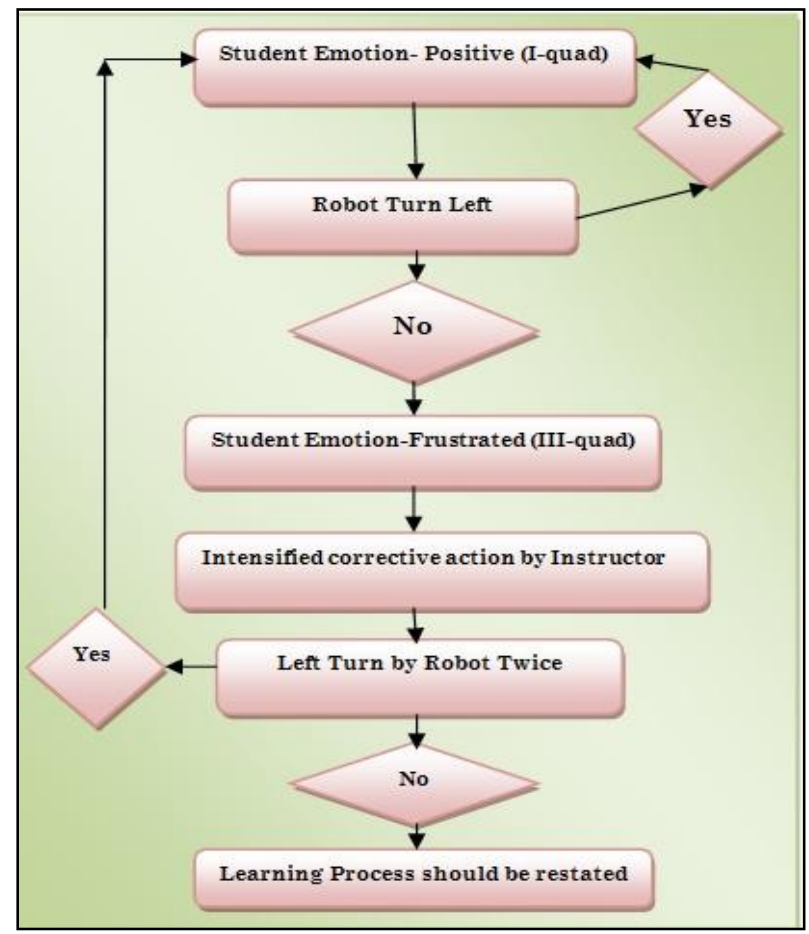

Figure 10. Flow chart showing the corrective measures using robotic actions [1]

\section{MERITS}

Constructive learning using robots maintains a healthy learning rate by analyzing the various emotions and necessary corrective actions. Robotic movements help the tutor to increase the learning rate and to verify the responses from the student. This learning increases the overall efficiency, effectiveness, interaction, observations and interest under the strict monitoring of the robot. Current robotic system can be used to monitor and to recognize the emotions of the mentally retarded children.

\section{EFFECTIVENESS OF DIFFERENT MEDIAS}

Table 1: Analysis of effectiveness of different Medias

\begin{tabular}{|c|c|}
\hline $\begin{array}{l}\text { DIFFERENT } \\
\text { MEDIAS IN } \\
\text { LEARNING }\end{array}$ & $\begin{array}{c}\text { EFFECTIVENESS } \\
\text { OF LEARNING }\end{array}$ \\
\hline Books & Moderate \\
\hline Audio Tapes & Less \\
\hline $\begin{array}{l}\text { Video Based } \\
\text { Interaction }\end{array}$ & Moderate \\
\hline $\begin{array}{c}\text { Constructive Learning } \\
\text { Robot }\end{array}$ & high \\
\hline
\end{tabular}

\section{CHALLENGES AND ISSUES}

Emotion analysis is the important part in the constructive learning using robots. Wrong facial expressions can mislead the robotic movements by creating errors and make the instructor's task more complex. According to the current development scenario, a robot can only imitate a single instructor inside the class. It also monitors and recognizes the emotions of a single student at a time.

\section{CONCLUSION}

Constructive learning incorporates positive learning to enhance the learning rate. Robot will act as monitoring system inside the class to maintain a healthy learning rate. Student's emotions are analyzed through facial expressions for classifying them into positive or negative emotions. Once the emotions are analyzed, necessary movements are provided by the robot so that instructor can make intensified actions. But the current situation allows only humans to activate the intensified corrective action.

\section{FUTURE SCOPE}

Action units can be modified to incorporate more number of facial expressions. Verification should be done to check whether the emotions recognized are genuine or not. Since a class room contains a large number of students, monitoring should be extent to all the students inside the class. Robots should be capable of identifying, classifying and recognizing the emotions of all students and should provide guidance to the instructor to improve the learning rate. Programming should be done in such a way that makes robot capable of imitating more number of teachers inside the class room. In future, a robotic 
system can be developed that can analyze more real time facial expressions and perform intensified corrective actions by replacing the human teachers.

\section{REFERENCES}

[1] Amarjot Singh, Sri Krishna Karanam,Devinder kumar, 2013."Constructive Learning for Human Robot Interaction", In IEEE Journals and Magazines Vol 32,Issue 4,pp13-19

[2] J. Han, M. Jo, S. Park, and S. Kim, 2005. "The educational use of home robots for children," in Proc. IEEE Int. Workshop Robot and Human Interactive Communication (ROMAN 2005), , pp. 378-383.

[3] Fumio Hara, 2004."Artificial Emotion of Face Robot through Learning in Communicative Interactions with Human", in Proc. IEEE Int Workshop Robot and Human Interactive Communication, 7-15

[4] R. Murphy, T. Nomura, A. Billard, and J. Burke, 2010."Human-robot interaction," IEEE Robot. Automat Mag., vol. 17, pp. 85-89.

[5] B. Kort, R Reilly, and R. W. Picard, 2001. "An effective model of interplay between emotions and learning: Reengineering educational pedagogy-Building a learning companion," in Proc. IEEE Int. Conf. Advanced Learning Technologies, pp. 43-46.

[6] Y. Yamada, Y. Hirawawa, S. Huang, Y. Umetani, and K. Suita,1997." Human - Robot Contact in the Safeguarding Space," IEEE/ASME Transactions on Mechatronics, vol. 2, pp. 230-236.

[7] Y. Yamada, T. Yamamoto, T. Morizono, and Y. Umetani,1999. "FTA Based Issues on Securing Human Safety in a Human Robot Coexistence System," presented at IEEE Systems, Man and Cybernetics SMC'99.

[8] Kanda, T., Hirano, T., Eaton, D., \& Ishiguro, H., 2004. "Interactive Robots as Social Partners and Peer Tutors for Children: A Field Trial", Human Computer Interaction, vol. 19, 61 84.
[9] Kiesler S. \& Hinds P., 2004. "Introduction to This Special Issue on Human Robot Interaction", Human-Computer Interaction, vol. 19, pp.1 8.

[10] H. Taoand T. S. Huang, 1998 "Connected vibrations: A modal analysis approach to non-rigid motion tracking," in Proc. IEEE Conf. Computer Vision and Pattern Recognition, pp. 735-740.

[11] P. Ekman, 1992"An argument for basic emotions," Cogn. Emot., vol. 6, no. 3-4, pp. 169-300.

[12] Noriaki Mitsunaga, Christian Smith, Takayuki Kanda, Hiroshi Ishiguro, Norihiro Hagita, august 2008.” Adapting Robot Behavior for Human-Robot Interaction", IEEE transactions on robotics, vol. 24 , no. 4

[13] N. Najmaei and M. Kermani, 1999."Super quadric obstacle modeling and a danger evaluation method with K. Ikuta and M. Nokata, "General evaluation method of safety for human care robots," in Proc. IEEE Int. Conf. Robot. Autom.vol. 3,pp. 2065-2072.

[14] R. Bischoff and V. Graefe, 2004. "Hermes-A versatile personal robotic assistant," Proc. IEEE, vol. 92, no. 11, pp. 1759-1779.

[15] Scholtz, J., 2003“Theory and Evaluation of Human Robot Interactions", Proceeding of Hawaii International Conference on System Science, pp.36.

[16] Scholtz, J. \& Bahrami, S.,2004. "Human-Robot Interaction: Development of an Evaluation Methodology for the Bystander Role of Interaction", Proceeding of International System, Man, and Cybernetics Conference

[17] K. Ikuta and M. Nokata, 1999.“General evaluation method of safety for human care robots," in Proc. IEEE Int. Conf. Robot. Autom.vol. 3,pp. 2065-2072.

[18] http://www.intechopen.com/books/human-robotinteraction/robot-aided-learning-and-r-learning-services

[19] https://developer.valvesoftware.com/wiki/Character_Facia 1_Animation_Shapekey_Set

[20] http://www.femininebeauty.info/eyebrow-aesthetics 